\title{
An dentigerous variant of ameloblastoma in mandible - An unusual case report and review of literature.
}

\author{
Neha Goyal ${ }^{1}$, Ajay Kumar Pillai ${ }^{2}$, Swapnil Moghe ${ }^{2}$, Vineesh Vishnu ${ }^{3}$, \\ Saksham Nahar ${ }^{4}$ \\ ${ }^{l}$ Dental Surgeon.Government District Hospital ,Mungeli \\ ${ }^{2,2}$ Reader, Dept. Of Oral \& Maxillofacial Surgery, People's Dental Academy, Bhopal. \\ ${ }^{3} P G$ Dept of Public health Dentistry. PDA.Bhopal \\ ${ }^{4}$ PG. Dept. Of Oral \& Maxillofacial Surgery, People's Dental Academy, Bhopal.
}

\begin{abstract}
Ameloblastoma is a true neoplasm of odontogenic epithelial origin. It is the second most common odontogenic neoplasm, and only odontoma outnumbers it in reported frequency of occurrence. Its incidence, combined with its clinical behaviour, makes ameloblastoma the most significant odontogenic neoplasm. Unicystic ameloblastoma (UA) refers to those cystic lesions that show clinical, radiographic, or gross features of a mandibular cyst, but on histological examination, it showed a typical ameloblastomatous epithelium lining part of the cyst cavity, with or without luminal and/or mural tumor growth. It accounts for 5-15\% of all intraosseous ameloblastomas. We report a case of unicystic ameloblastoma in a 16-year-old female, and review the literature.
\end{abstract}

\section{Introduction}

Swellings of mandible mainly occur due to odontogenic or non-odontogenic etiology.Many benign lesions cause mandibular swellings, and these can be divided into those of odontogenic and nonodontogenic origin. Lesions include ameloblastoma, radicular cyst, dentigerous cyst, keratocystic odontogenic tumour, central giant cell granuloma, fibro-osseous lesions and osteomas ${ }^{1}$.

Ameloblastoma is a slow-growing, persistent, and locally aggressive neoplasm of epithelial origin. Its peak incidence is in the 3rd to 4th decades of life and has an equal sex distribution. It is often associated with an unerrupted third molar ${ }^{2}$. The vast majority of ameloblastomas arise in the mandible, and the majority of these are found in the angle and ramus region. There are three forms of ameloblastomas, namely multicystic, peripheral, and unicystic tumors ${ }^{3}$.

Multicystic ameloblastoma is the most common variety and represents $86 \%$ of cases. Peripheral tumors are odontogenic tumors, with the histological characteristics of intraosseous ameloblastoma that occur solely in the soft tissues covering the tooth-bearing parts of the jaws. Unicystic tumors include those that have been variously referred to as mural ameloblastomas, luminal ameloblastomas, and ameloblastomas arising in dentigerous cysts ${ }^{4}$.

In 1977, Robinson and Martinez, described as, the gross and microscopic features in an unicystic ameloblastoma, is associated with a large cystic cavity with either luminal or mural proliferation of ameloblastic tumor cells. They referred to this variant as unicystic ameloblastoma ${ }^{5}$. In 1984 Gardner and Corio reported a series of thirty-five instances of unicystic ameloblastoma exhibiting a plexiform pattern of cell proliferation ${ }^{6}$.

The goal of treatment in ameloblastoma is to achieve complete excision and appropriate reconstruction. We present a case of a large unicystic mandibular ameloblastoma in a 16 year old female.

\section{Case Report}

A 16 year old female, came up with the complaint s of swelling over her left face region since 7 years.

Patient was apparently alright 7 years ago, then she noticed a small swelling over her left lower jaw region for which she went for treatment, and underwent marsupialization w.r.t. 38 and extraction of 36, and was diagnosed by histopathologists as Dentigerous cyst w.r.t. unerrupted 38. At that time, she was relieved of the complaint. After three years, she again noticed a small sized swelling with the same region, which increased in size gradually in the duration of 4 years. There was no associated pain or discharge with the swelling. 


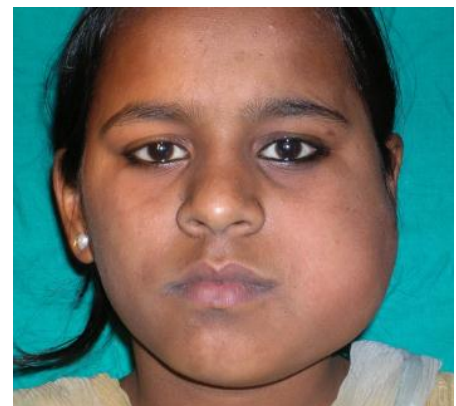

Figure 1: pre operative photograph

On physical examination (Fig. 1), it was found, a solitary diffused oval shaped swelling present over left middle \& lower $3^{\text {rd }}$ face region of size $6 \mathrm{~cm} \mathrm{X} \mathrm{5cm,} \mathrm{its} \mathrm{margins} \mathrm{were} \mathrm{diffused,} \mathrm{overlying} \mathrm{skin} \mathrm{was} \mathrm{normal.}$ On palpation swelling was firm with no associated tenderness and no raised overlying temperature.

On intra oral examination, it was found, lower anterior Vestibular obliteration, extending from 33-38 region, buccal cortical bone expansion was noted from 33 to 38. Also, lingually cortical plate expansion from 33-35 region was seen. Lingually displaced and tilted 37, with missing 36 was present. On palpation it was firm $\&$ multi lobular in consistency extending from 33 till ramus length with no tenderness (Fig. 2).

Systemic examination was normal, further investigations included orthopantomogram and CT scan with 3D reconstruction. Orthopantomogram (OPG) revealed a large cystic lesion in the left side of mandible (Fig. 3).

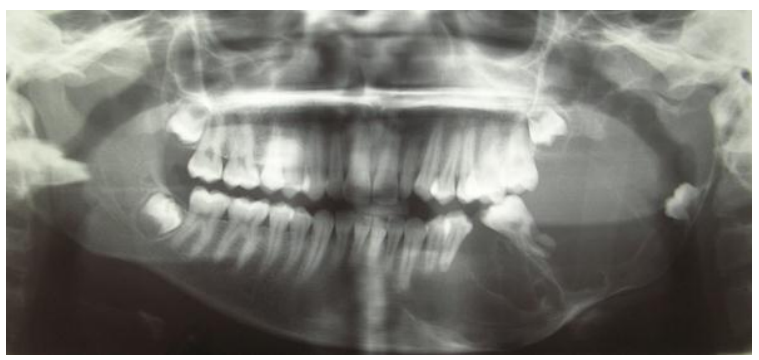

Figure 2: preoperative panoramic radiograph

CT scan \& 3D CT Reconstruction revealed a multilocular lesion extending from left lower canine till left half of mandible including coronoid and condyle process, with a thinned out cortex (Fig. 4).

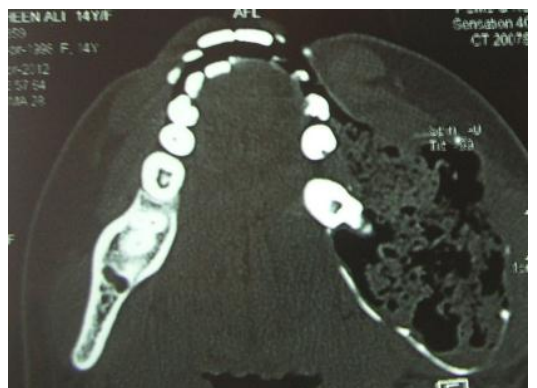

Figure 3: Axial CT view of the pathology with involving periosteum \& muscular layer

Further, incisional biopsy of the lesion was performed and the histopathological impression was given of unicystic ameloblastoma, intraluminal and mural type (Fig. 5).

Later a systemic surgical planning and evaluation was done and patient was taken up for surgery under general anaesthesia. A hemi mandibulectomy was done via a extended submandibular incision, reconstruction was done using titanium reconstruction plate and anterior ileac cortico-cancellous bone graft (Fig. 6), and primary closure achieved. The resected specimen (Fig. 7) was subjected to radiographic examination (Fig. 8) and later it was sent for histopathological analysis and was given the diagnosis of unicystic ameloblastoma. 


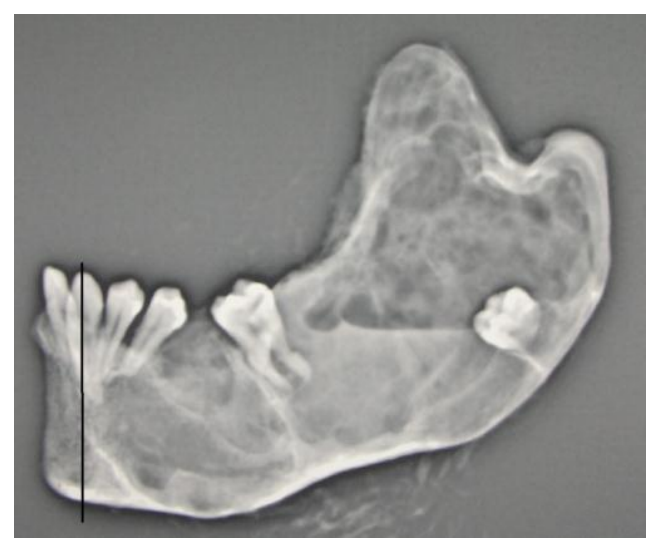

Figure 4: radiographic examination of resected specimen

Patent was on constant follow up for two years with out any clinical or radiological sign of recurrence. Latter on patient was delivered a RPD and is recalled for follow up every six mon .

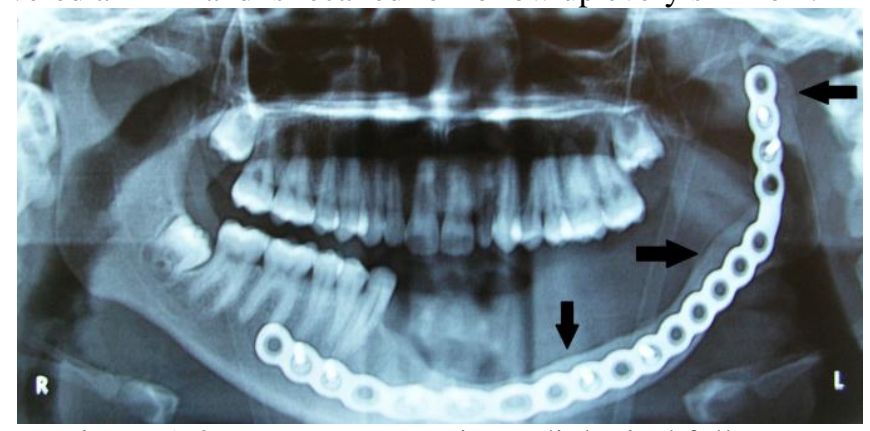

Figure 5: 2 years Post operative radiological follow up

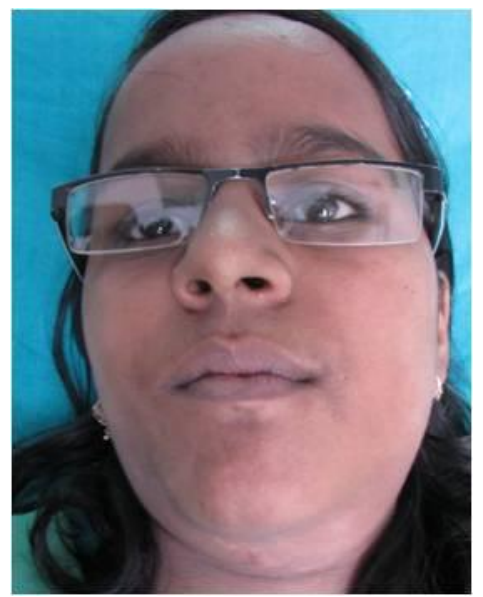

Figure 6: 2 years Post operative clinical follow up.

\section{Discussion}

Unilocular ameloblastoma (UA) is a rare type of ameloblastoma, accounting for about $6 \%$ of ameloblastomas. It usually occurs in a younger age group, with about $50 \%$ of the cases occurring in the second decade of life. More than $90 \%$ are located in the mandible ${ }^{3,7,8 .}$ Between $50 \%$ and $80 \%$ of cases are associated with tooth impaction, the mandibular third molar being most often involved. The 'dentigerous' type occurs 8 years earlier on average than the 'non-dentigerous' variant. Patients most commonly present with swelling and facial asymmetry, pain being an occasional presenting symptom. Mucosal ulceration is rare, but may be caused by continued growth of the tumor. Small lesions are sometimes discovered more on routine radiographic screening examinations or as a result of local effects (like tooth mobility, occlussal alterations and failure of eruption of teeth) produced by the tumor ${ }^{9}$. 
In the case we present, the patient first presented with dentigerous cyst with the impacted 3rd molar which was treated by marsupialization. Later the cystic lining of dentigerous cyst transformed into unicystic ameloblastoma in the duration of 4 years and presented, such a devastating condition.

The radiological features of ameloblastoma resembles to many odontogenic and non odontogenic cyst in the jaws. The bone is expanded, appears cystic resembling a honeycomb or soap bubble and is eccentrically ballooned. There may be destruction or perforation of the cortex and a periosteal reaction may be evident.It may appear unilocular or multilocular radiolucent, radiopaque or mixed ${ }^{10}$.

Histologically, the minimum criterion for diagnosing a lesion as UA is the demonstration of a single cystic sac lined by odontogenic (ameloblastomatous) epithelium often seen only in focal areas. UA should be differentiated from odontogenic cysts because the former has a higher rate of recurrence than the latter ${ }^{11}$. In a clinicopathologic study of 57 cases of unicystic ameloblastoma, Ackermann ${ }^{3}$ classified this entity into the following three histologic groups:-

Group I: Luminal UA (tumor confined to the luminal surface of the cyst)

Group II: Intraluminal/plexiform UA (nodular proliferation into the lumen without infiltration of tumor cells into the connective tissue wall), and

Group III: Mural UA (invasive islands of ameloblastomatous epithelium in the connective tissue wall not involving the entire epithelium).

Another histologic sub grouping by Philipsen and Reichart ${ }^{11}$ has also been described:-

Subgroup 1: Luminal UA

Subgroup 1.2: Luminal and intraluminal

Subgroup 1.2.3: Luminal, intraluminal and intramural

Subgroup 1.3: Luminal and intramural

The UAs diagnosed as subgroups 1 and 1.2 can be treated conservatively (careful enucleation), whereas subgroups 1.2.3 and 1.3 showing intramural growths require treated radical resection, as for a solid or multicystic ameloblastoma ${ }^{11}$.

The case we present comes under subgroup 1.3 and accordingly the radical treatment followed by reconstruction was undertaken for the patient.

Following enucleation, vigorous curettage of the bone should be avoided as it may implant foci of ameloblastoma more deeply into bone. Chemical cauterization with Carnoy's solution is also advocated for subgroups 1 and 1.2. Subgroups 1.2.3 and 1.3 have a high risk for recurrence, requiring more aggressive surgical procedures. This is because the cystic wall in these cases has islands of ameloblastoma tumor cells and there may be penetration into the surrounding cancellous bone ${ }^{12}$. Late recurrence following treatment is commonly seen, the average interval for recurrence being 7 years. Recurrence is also related to histologic subtypes of UA, with those invading the fibrous wall having a rate of $35.7 \%$, but others only $6.7 \%{ }^{13}$. Recurrence rates are also related to the type of initial treatment. Lau et $\mathrm{al}^{14}$ reported recurrence rates of $3.6 \%$ for resection, $30.5 \%$ for enucleation alone, $16 \%$ for enucleation followed by Carnoy's solution application, and $18 \%$ by marsupialization followed by enucleation (where the lesion reduced in size).

In a recent study conducted by Dhanuthai $\mathrm{K}$ et $\mathrm{al}^{15}$, the mean age of the patients diagnosed with ameloblastoma was $38.27 \pm 17.78$ years; $51.36 \%$ were men. Mandible $(84.26 \%)$ outnumbered maxilla and other locations combined in all countries. The number of multilocular radiolucencies $(43.40 \%)$ was comparable with that of unilocular radiolucencies $(42.04 \%)$. Follicular pattern was the most common histopathologic pattern (27.70\%), followed by plexiform (21.10\%) and unicystic pattern (20.71\%), respectively.

\section{Conclusion}

The diagnosis of unicystic/ multicystic ameloblastoma was based on clinical, histopathologic and CT features. Radiographically, most of ameloblastomas show multilocularity, whereas unilocular ameloblastomas show a single large unilocular radiolucency

Ameloblastoma is a tumor with a strong propensity for recurrence, especially when the ameloblastic focui penetrates the adjacent tissue from the wall of the cyst. The ability to predict this potential occurrence prior to surgery would greatly enhance therapeutic strategies for reducing the incidence.

Hence, the Pathologist should examine the tissue sections carefully in an attempt to determine whether ameloblastoma has penetrated the wall of the cyst or not so that the, possible treatment modification can be done and complications can be minimized. 


\section{References}

[1]. Kahairi A, Ahmad RL, Wan Islah L, Norra H: Management of large mandibular ameloblastoma - a case report and literature reviews; Archives of Orofacial Sciences 2008, 3(2):52-55.

[2]. Gerzenshtein J, Zhang F, Caplan J, Anand V, Lineaweaver W: Immediatemandibular reconstruction with microsurgical fibula flap transfer following wide resection for ameloblastoma. J Craniofac Surg 2006, 17(1):178-182.

[3]. Philipsen HP, Reichart PA: Classification of odontogenic tumors and allied lesions. Odontogenic tumors and allied lesions Quintessence Pub. Co. Ltd 2004, 21-3.

[4]. Chana, Jagdeep S, Yang-Ming Chang, Wei, Fu-Chan, Shen, Yu-Fen, Chan Chiu-Po, Lin Hsiu-Na, Tsai Chi-Ying, Jeng SengFeng: Segmental mandibulectomy and immediate free fibula osteoseptocutaneous flap reconstruction with endosteal implants: An ideal treatment method for mandibular ameloblastoma. Plast Reconstr Surg 2004, 113(1):80-87.

[5]. Robinson L, Martinez M. Unicystic ameloblastoma. Cancer 1977; $40: 2278-85$.

[6]. Gardner DG. Plexiform unicystic ameloblastoma. A diagnostic problem in dentigerous cysts. Cancer 1981; $47: 1358-63$.

[7]. Pizer ME, Page DG, Svirsky JA: Thirteen-year follow-up of large recurrent unicystic ameloblastoma of the mandible in a 15year-old boy. J Oral Maxillofac Surg 2002, 60:211-5.

[8]. Navarro CM, Principi SM, Massucato EM, Sposto MR: Maxillary unicystic ameloblastoma. Dentomaxillofac Radiol 2004, 33:60-2.

[9]. Roos RE, Raubenheimer EJ, van Heerden WF: Clinico-pathological study of 30 unicystic ameloblastomas. J Dent Assoc S Afr 1994, 49:559-62.

[10]. Moghe S, Saini N, Pillai A et al. Aneurysmal Bone Cyst Plus In an 8 Year Old Female, A Case Report. IOSR-JDMS 2013;13(4): 63-68.

[11]. Konouchi H, Asaumi J, Yanagi Y, Hisatomi M, Kawai N, Matsuzaki H, Kishi K: Usefulness of contrast enhanced-MRI in the diagnosis of unicystic ameloblastoma. Oral Oncol 2006, 42:481-6.

[12]. Philipsen HP, Reichart PA: Unicystic ameloblastoma. Odontogenic tumors and allied lesions London: Quintessence Pub. Co. Ltd 2004, 77-86.

[13]. Li T, Wu Y, Yu S, Yu G: Clinicopathological features of unicystic ameloblastoma with special reference to its recurrence. Zhonghua Kou Qiang Yi Xue Za Zhi 2002, 37:210-2.

[14]. Lau SL, Samman N: Recurrence related to treatment modalities of unicystic ameloblastoma: A systematic review. Int J Oral Maxillofac Surg 2006, 35:681-90.

[15]. Dhanuthai K, Chantarangsu S, Rojanawatsirivej S, Phattarataratip E, Darling M, Jackson-Boeters L, Said-Al-Naief N, Shin HI, An CH, Hong NT, An PH,Thosaporn W, Lam-Ubol A, Subarnbhesaj A : Ameloblastoma: a multicentric study. Oral Surg Oral Med Oral Pathol Oral Radiol. 2012 Jun;113(6):782-8. 